\title{
Postoperative pain and analgesic consumption after endoscopic and microscopic ear procedures
}

\author{
Richard Salzman, Tomas Bakaj, Ivo Starek
}

\begin{abstract}
Objective. Endoscopic ear surgery (EES) is considered a minimally invasive technique. It is hypothesized that patients after endoscopic procedures experience less pain and require fewer painkillers. Our aim was to compare patients' postoperative pain and need for analgesics in patients undergoing microscopic and endoscopic ear surgeries.

Methods. a single-institution, prospective study included 92 patients undergoing ear procedures (48 cholesteatoma and 44 reconstructive);(43 endoscopic and 49 microscopic) during 1/2016-1/2018. The postoperative pain (visual analogue scale (VAS) 0-10) and painkillers consumption were recorded and compared in each subgroup.

Results. VAS $(P=0.02)$ and analgesics consumption $(P=0.06)$ were lower after endoscopic ear surgeries.

In the cholesteatoma group, $94 \%$ of endoscopic patients reported VAS 0-2 in contrast to $58 \%$ of microscopic patients on day $1(P=0.04)$. Similar tendencies were revealed in reconstructive cases, i.e. $92 \%$ compared to $73 \%(P>0.05)$.

On day $1,92 \%$ of endoscopic, and $77 \%$ of microscopic reconstruction patients received no painkillers group ( $P=0.06)$. In the cholesteatoma patients, $88 \%$ of endoscopic patients, $43 \%$ of microscopic patients using endaural approach, and $75 \%$ of microscopic patients using retroauricular approach, required no painkillers on the postoperative day $1(P>0.05)$. Conclusions. Our study revealed that the EES is associated with less postoperative pain than the conventional microscopic ear surgery. It seems that it is rather the transmeatal approach, and not the endoscope itself that reduces postoperative pain. The removal of the same size cholesteatoma using endoscope (rather than using microscope) leads to less intensive postoperative pain. Consequently, it was not surprising that patients after endoscopic ear surgery very rarely took painkillers.
\end{abstract}

Key words: life quality, pain measurement, pain, stapes surgery, transcanal endoscopic ear surgery, tympanoplasty

Received: September 13, 2020; Revised: January 12, 2021; Accepted: January 12, 2021; Available online: February 4, 2021

https://doi.org/10.5507/bp.2021.006

(c) 2022 The Authors; https://creativecommons.org/licenses/by/4.0/

Department of Otorhinolaryngology and Head and Neck Surgery, Faculty of Medicine and Dentistry, Palacky University Olomouc and University Hospital Olomouc, I. P. Pavlova 6, 775 20, Olomouc, Czech Republic

Corresponding author: Richard Salzman, e-mail: richard.salzman@fnol.cz

\section{INTRODUCTION}

Since the 1950's, tympanoplasty has been performed using microscope through retroauricular, endaural or transmeatal approach. As a microscopic view is limited by the narrowest portion of the ear canal, the transmeatal approach is applicable only in a small number of patients. Therefore, the former two ways to enter relevant temporal bone spaces remain the mainstay approach worldwide.

Endoscopic ear surgery has become more popular recently. The major difference between the endoscopic and microscopic tympanoplasty is a surgical view. Endoscopes provide a closer, wider, and even angled view, allowing thus better visualization of middle ear structures and better comprehension of middle ear anatomy and physiology. Therefore, the transcanal endoscopic ear surgery (TEES) is being performed via ear canal and avoids unnecessary external incisions, dissection and drilling in healthy tissues on the access route to the pathology ${ }^{1}$.

TEES is generally accepted as a minimally invasive technique ${ }^{2}$. It can be, therefore, hypothesized that patients after endoscopic ear procedures could experience less pain and require fewer painkillers.
Our objective was to compare patients' quality of life in regards to postoperative pain and need for analgesics in patients undergoing microscopic and endoscopic ear surgeries.

\section{PATIENTS AND METHODS}

This single-institution, prospective study was conducted between January 2016 and January 2018 at the Department of Otorhinolaryngology and Head and Neck Surgery, Palacky University and University Hospital Olomouc, Czech Republic after approval by the Ethics Review Board of Palacký University, Olomouc.

In total 92 consecutive patients undergoing ear procedures were included after they signed the informed consent form. Patients not capable of general anesthesia and those with chronic pain were excluded from the study. Additionally, patients planned for primary meato/ canalplasties; or cholesteatomas with mastoid involvement on a CT scan were excluded as well because bone removal or dissection in the ear canal could increase postoperative pain and bias our study. All procedures were performed by two experienced consultant otologists. 
Patient cohort was divided into cholesteatoma and reconstruction groups (myringo-/ossiculo-/stapedoplasties). These were subdivided into endoscopic and microscopic cases. Endoscopically assisted microscopic procedures were considered microscopic since the endoscopes were used solely as an adjunct for improved visualization of difficult access sites or limited dissection in these areas (class 1 and 2 a according to Cohen's classification ${ }^{3}$ ).

In TEES, using 0 and/or 30 degree, $2.7 \mathrm{~mm}$ diameter and $11 \mathrm{~cm}$ long rigid endoscopes attached to Karl Storz LED 175 light source (set to 30\% intensity), we applied the same surgical technique as in the traditional microscopic surgery. TEES differed from the endaural (32 cases) or retroauricular (17 cases) microscopic approaches in the location of incision in the ear canal. In all patients, the ear canal was thoroughly infiltrated using 1-2 $\mathrm{mL}$ of solution (prepared from $10 \mathrm{~mL}$ of $1 \%$ mesocaine with 3 drops of 1/1000 epinephrine). In endaural and retroauricular incisions, an additional $5 \mathrm{~mL}$ of the same solution was used. The meatal hair wasn't trimmed in any patient as this is a common practice of the team. The elevation of a wide tympano-meatal flap was achieved using Rosen knife (with integrated suction) and cottonoids soaked in 1/10 000 epinephrine.

Pain intensities were recorded using a visual analogue scale (VAS) anchored to "no pain at all" on 0 mark and "worst pain I can imagine" marked 10. Since postoperative day 1, each patient was daily approached by a nurse or a surgeon and asked about the VAS of pain. The given analgesics were recorded. After discharged from the hospital, patients recorded painkillers consumption and VAS themselves.

For each postoperative day, we calculated the number of patients who didn't ask for any painkillers on that particular day.

All patients received the premedication of $10 \mathrm{mg}$ of benzodiazepine 30 min prior to surgery as is the common practice in the Czech Republic. Perioperatively sufentanil was administered. Early postoperatively an intravenous bolus of $2-5 \mathrm{~mL}$ of metamizole $(500 \mathrm{mg} / \mathrm{mL})$ was applied to all patients. Patients requiring additional analgesia later on were given metamizole $(500 \mathrm{mg})$ perorally. A rescue medication was decided to be paracetamol 1000 mg which was prescribed to a single patient after microscopic stapes surgery.

When comparing patients graded according to STAM classification $^{4}$ (in cholesteatoma group), we included only patients undergoing primary cholesteatoma removal.

All statistical analyses were performed using Dell Statistica (data analysis software system), version 13. A $P$-value of 0.05 was considered to be statistically significant.

\section{RESULTS}

\section{Cohort characteristics}

A total of 92 patients ( 49 males, 43 females) were included. Patients' age ranged from 15-78 (mean $46.7 \pm$ 16.5 years). The left to right ear ratio was $49: 43$. Patient
Table 1. Patient characteristics and types of procedures in each particular subgroup.

\begin{tabular}{lcc}
\hline & $\begin{array}{c}\text { Endoscope } \\
\mathrm{n}=43\end{array}$ & $\begin{array}{c}\text { Microscope } \\
\mathrm{n}=49\end{array}$ \\
\hline Cholesteatoma group & 17 & 31 \\
Age (mean \pm SD) & $42.8 \pm 16.1 \mathrm{yrs}$ & $42.9 \pm 20.2 \mathrm{yrs}$ \\
Age (range) & $15-69 \mathrm{yrs}$ & $16-78 \mathrm{yrs}$ \\
- Tympanoplasty & 14 & 20 \\
- $2^{\text {nd }}$ look tympanoplasty & 4 & 10 \\
Reconstruction group & 25 & 19 \\
Age (mean \pm SD) & $49.6 \pm 12.1 \mathrm{yrs}$ & $52.5 \pm 12.6 \mathrm{yrs}$ \\
Age (range) & $23-70 \mathrm{yrs}$ & $31-71 \mathrm{yrs}$ \\
- Myringoplasty & 11 & 8 \\
- Ossiculoplasty & 1 & 2 \\
- Stapes surgery & 13 & 9 \\
\hline
\end{tabular}

$\mathrm{SD}$, standard deviation

characteristics and types of procedures in each particular subgroup are described in Table 1.

\section{Visual analogue scale of pain intensity}

Comparing VAS on day 1 of all endoscopic and microscopic patients showed that patients after endoscopic surgeries suffered less pain (Mann-Whitney, $P=0.02$ ).

Then we focused on the cholesteatoma group. On the postoperative day 1, 29\% of endoscopic patients reported VAS $0,41 \%$ reported VAS 1, 24\% VAS 2 and 6\% VAS 4. On the contrary, on day 1 after microscopic procedures, $19 \%$ of patients reported VAS $0,26 \%$ VAS $1,13 \%$ VAS 2 , $13 \%$ VAS 3, 10\% VAS 4, 10\% VAS 5, 6\% VAS 6 and 3\% VAS 8. Endoscopic cholesteatoma patients suffered from less pain (Mann-Whitney, $P<0.05$, Fig. 1).

In order to reduce a risk of bias selection, we compared VAS in subgroups graded according to STAM classification ${ }^{4}$. Stage 1 cholesteatomas $(n=17,11$ endoscopic vs. 6 microscopic cases) showed less intensive postoperative pain in endoscopic group (Mann-Whitney, $P=0.02$, Fig. 3). Stage 2 cholesteatomas $(n=13$, out of them only 4 in endoscopic group) showed only similar tendency without statistical significance $(P=0.71)$.

The comparison of reconstruction cases revealed similar tendencies with no statistical significance. On the postoperative day 1, 36\% of endoscopic group reported VAS $0,28 \%$ reported VAS $1,28 \%$ VAS 2 and $8 \%$ VAS 3 . On the contrary, on day 1 after microscopic procedures, $31 \%$ of patients reported VAS 0, 31\% VAS 1, 11\% VAS 2, 16\% VAS 3 and 11\% VAS 5 (Fig. 2).

\section{Analgesic consumption}

Consumption of analgesics in the reconstruction group, as reported in the Fig. 4, shows that $92.0 \%$ of endoscopic patients, and only $76.5 \%$ of microscopic patients (using endaural approach), did not receive any painkillers on the first postoperative day, respectively $100.0 \%$ and $82.4 \%$ on the second postoperative day. The comparison shows strong tendency towards smaller need for painkillers in the endoscopic subgroup ( $P=0.06, \mathrm{chi}^{2}$ test). There 


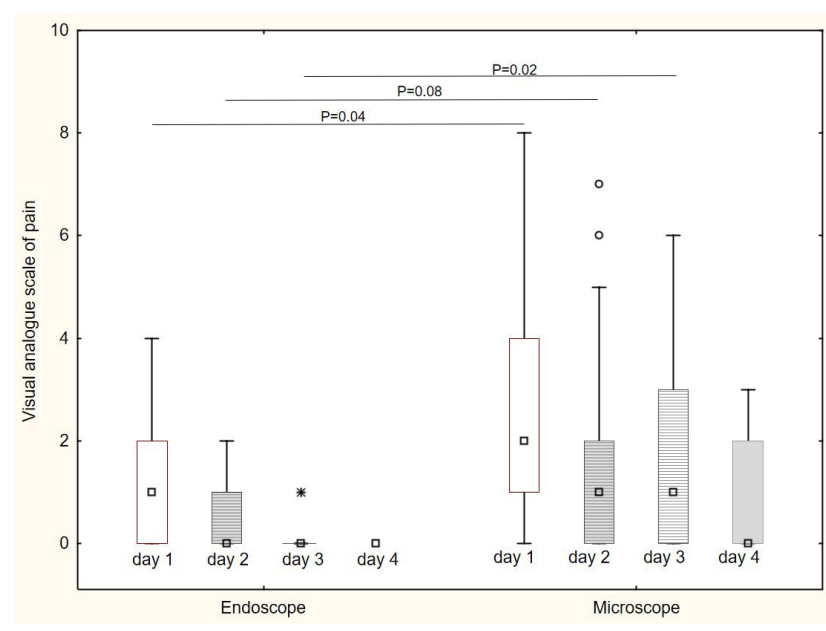

Fig. 1. A comparison of visual analogue scale of pain intensity after endoscopic and microscopic ear procedures in cholesteatoma group.

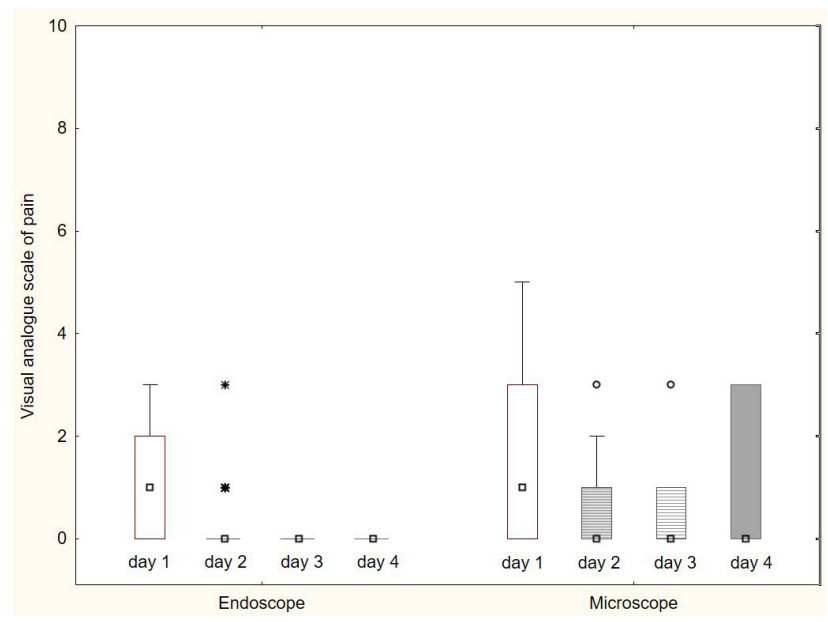

Fig. 2. A comparison of visual analogue scale of pain intensity after endoscopic and microscopic ear procedures in reconstruction group.

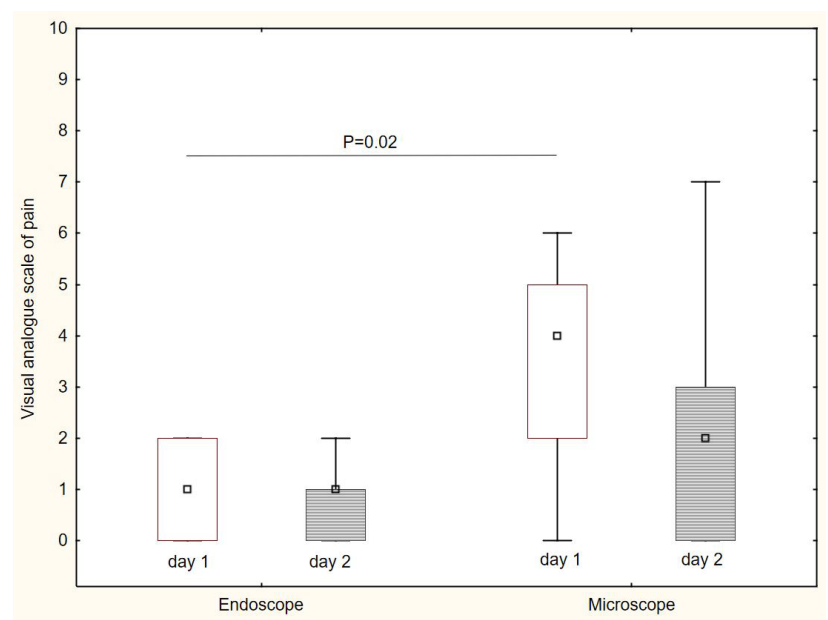

Fig. 3. A comparison of visual analogue scale of pain intensity after endoscopic and microscopic ear procedures in grade 1 cholesteatoma subgroup. was no retroauricular approach used in this subgroup. Two patients underwent transmeatal approach using microscope. These 2 patients required absolutely no painkillers.

Fig. 5 shows similar tendencies in cholesteatoma cases ( $P>0.05$, chi $^{2}$ test). In total $87.5 \%$ of endoscopic patients, $42.9 \%$ of microscopic patients using endaural approach, and $75.0 \%$ of microscopic patients using retroauricular approach, required no painkillers on the first postoperative day, respectively $93.8 \%, 64.3 \%$, and $85.7 \%$ on the second postoperative day.

\section{DISCUSSION}

Although almost 3 decades have elapsed since the first transcanal use of endoscope during ear surgery ${ }^{5}$, the TEES has gained wider acceptance only in recent years. The rationale of this procedure has been described elsewhere ${ }^{6}$. TEES is generally considered to be a minimally invasive technique ${ }^{7}$. Thus, it could be hypothesized that it improves patients' postoperative comfort when compared with the traditional microscopic procedures. Even though the ear surgeries are not usually associated with major postoperative pain, further suppression of pain and reduced need for painkillers would be appreciated by patients and could reduce treatment costs and expedite discharge to home care. Surprisingly, very little attention has been, so far, paid to this aspect of TEES in the published literature.

Our literature review identified few papers looking into the postoperative pain after ear surgery. The objective of papers by Guntinas-Lichius was to create and validate a tool which would allow better pain management after ENT procedures including ear surgeries ${ }^{8-9}$. His questionnaire is in German, therefore, not generally applicable. Sommer compared postoperative pain after various ENT procedures using VAS. He concluded that less than 20 per cent of patients after an ear surgery complained of pain reported as VAS $\geq 4$ in a scale 0 to $10\left(\right.$ ref. $\left.{ }^{10}\right)$. Our study revealed corresponding figures.

Only recently, a single paper by Kakehata ${ }^{11}$ presented a direct comparison of TEES and microscopic approach. Our results support his findings that TEES is associated with less postoperative pain, lower consumption of nonsteroidal painkillers. Interestingly, he found that extent of bone removal seems to play less important role than presence of external incision.

Our results indicate that TEES leads to lower postoperative pain than traditional microscopic procedures.

One could hypothesize that the transmeatal microscopic approach would reveal similar advantage when compared to endaural or retroauricular approaches using microscope. Unfortunately, no study showing this comparison could be identified. Despite having only a small number of transmeatal microscopic cases, our study supports the idea that it is rather the transmeatal approach and not the endoscope itself that reduces postoperative pain (Fig. 4). 


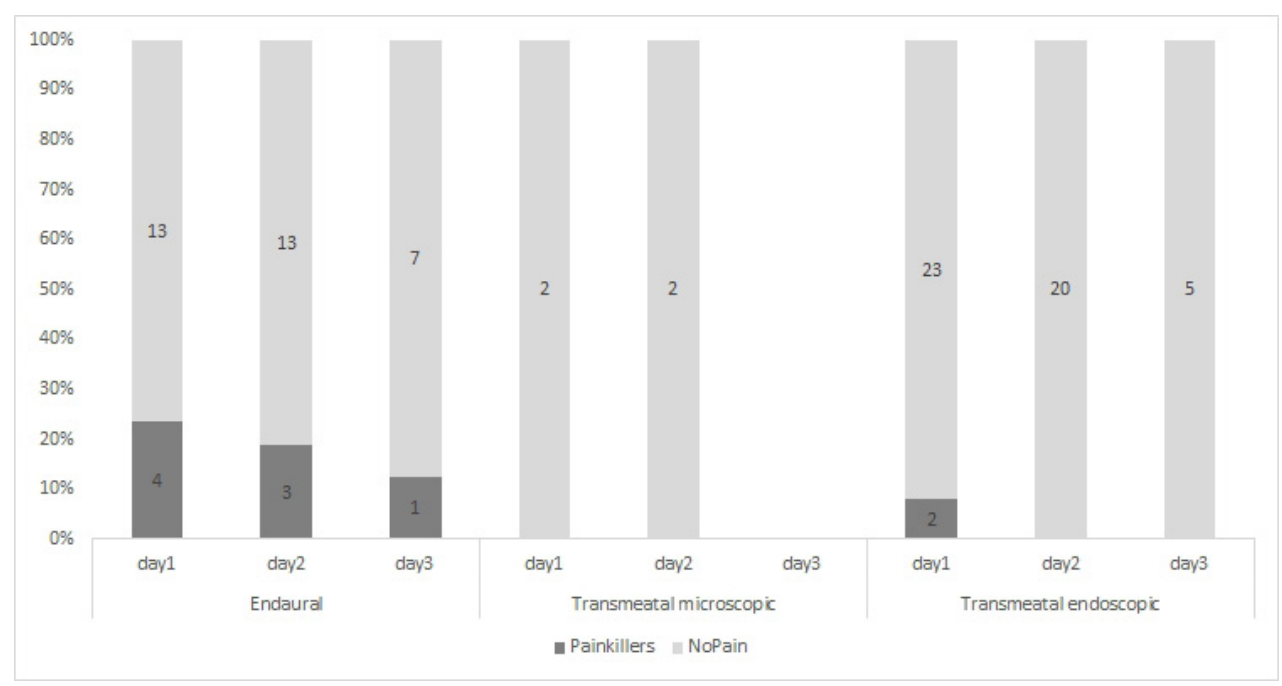

Fig. 4. A consumption of analgetics after endoscopic and microscopic ear surgeries in the reconstruction group only. The graph reports the number of patients taking any painkiller versus patients not requiring any pain control.

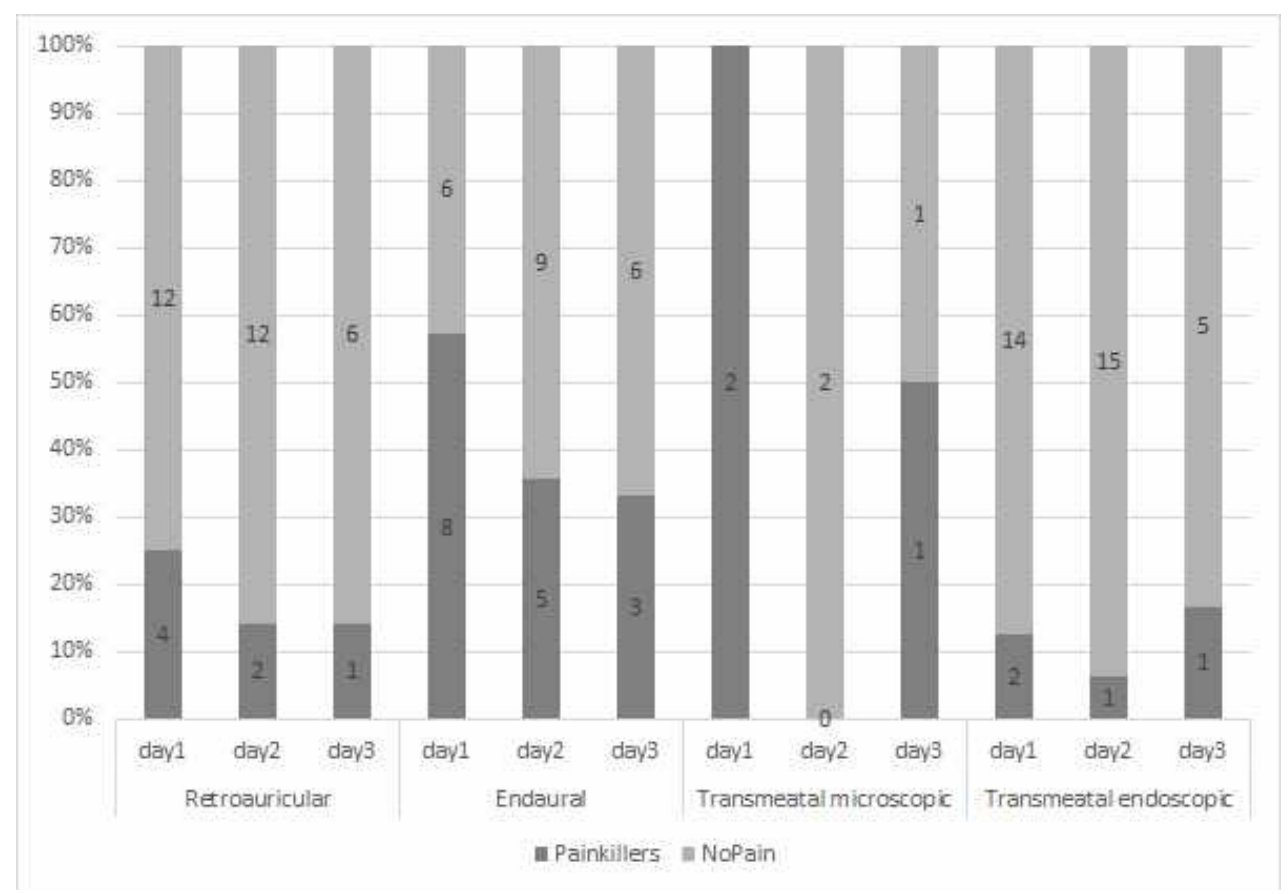

Fig. 5. A consumption of analgetics after endoscopic and microscopic ear surgeries in the cholesteatoma group only. The graph reports the number of patients taking any painkiller versus patients not requiring any pain control.

It is generally accepted that the less radical endoscopic technique offers close-up and angled view and, therefore, requires less removal of healthy structures ${ }^{12}$. Endoscopes offering sufficient access and view via transmeatal approach, therefore, allow more surgeries to be performed transmeatally.

We believe that TEES is associated with less postoperative pain as it uses shorter incision via transmeatal approach, less soft tissue dissection en route to the "unhealthy" region of interest, due to better visualization of operative field even behind the corner.
The decreased postoperative pain is tightly related to the reduced painkiller need. Not only it saves hospital staff workload when giving out analgesia in a ward, but it also lowers costs for painkillers as such.

The weakness of the study presented is a selection bias. Extensive cholesteatomas are more likely to require retroauricular or endaural approach. These approaches use longer incisions and, therefore, are likely to lead to more pain postoperatively. In order to reduce the effect of this selection bias, we compared subgroups of patients graded according to STAM classification ${ }^{4}$. The compari- 
son of these better comparable samples confirmed that removal of the same size disease using endoscope leads to less intensive postoperative pain. Furthermore, the low patient numbers often only showed tendencies and not statistically significant differences. Even though our figures were often strongly suggestive of clear differences, the calculations did not reveal significance due to low numbers of cases in individual subgroups (for example with $P=0.06$ ).

\section{CONCLUSIONS}

Our study clearly suggests that the transcanal endoscopic ear surgery is associated with less postoperative pain than the conventional microscopic ear surgery. It seems that it is rather the transmeatal approach, and not the endoscope itself that reduces postoperative pain. The removal of the same size cholesteatoma using endoscope (rather than using microscope) leads to less intensive postoperative pain. Consequently, it was not surprising that the patients after TEES very rarely took painkillers to control their postoperative pain.

\section{ABBREVIATIONS}

ENT, Ear nos throat; TEES, Transcanal endoscopic ear surgery; VAS, Visual analogue scale.

Acknowledgement: The research was funded from the internal grant of the Palacký University Olomouc IGA LF 2020-27 and by the Ministry of Health of the Czech Republic RVO FNOL 00098892.

Author contributions: RS: study conception and design, surgeon, manuscript preparation, final revision; TB: surgeon, manuscript revision; IS: design, final revision. All authors read and approved the final manuscript.

Conflict of interest statement: None declared. Ethics approval and consent to participate: All procedures performed in studies involving human participants were in accordance with the ethical standards of the institutional and/or national research committee and with the
1964 Helsinki Declaration and its later amendments or comparable ethical standards. The study was approved by the Ethics Review Board of Palacký University, Olomouc (\#120/18 on July 9, 2018). Written informed consent was obtained from all individual participants included in the study.

\section{REFERENCES}

1. Salzman R. Endoscopic ear surgery - review. Otorinolaryng a Foniat /Prague/ 2016;65(3):184-87.

2. Badr-el-Dine M. Value of ear endoscopy in cholesteatoma surgery. Otol Neurotol 2002;23(5):631-35.

3. Cohen MS, Basonbul RA, Barber SR, Kozin ED, Rivas AC, Lee DJ.Development and validation of an endoscopic ear surgery classification system. Laryngoscope 2018;128(4):967-970. doi: 10.1002/ lary. 26802

4. Yung $M$, Tono $T$, Olszewska, Yamamoto $Y$, Sudhoff $H$, Sakagami $M$, Mulder J, Kojima H, İncesulu A, Trabalzini F, Özgirgin N. EAONO/JOS joint consensus statements on the definitions, classification and staging of middle ear cholesteatoma. J Int Adv Otol 2017;13(1):1-8. doi: 10.5152/iao.2017.3363

5. Thomassin JM, Korchia D, Doris JM. Endoscopic-guided otosurgery in the prevention of residual cholesteatomas. Laryngoscope 1993;103(8):939-43. doi: abs/10.1288/00005537-199308000-00021

6. Tarabichi M, Marchioni D, Presutti L, Nogueira JF, Pothier D. Endoscopic transcanal ear anatomy and dissection. Otolaryngol Clin North Am 2013;46(2):131-54. doi: 10.1016/j.otc.2013.02.001

7. Presutti L, Gioacchini FM, Alicandri-Ciufelli M, Villari D, Marchioni D.Results of endoscopic middle ear surgery for cholesteatoma treatment: a systematic review. Acta Otorhinolaryngol Ital 2014;34(3):153-57.

8. Guntinas-Lichius O, Volk GF, Geissler K, Komann M, Meissner W. Pain after pediatric otorhinolaryngologic surgery: a prospective multicentre trial. Eur Arch Otorhinolaryngol 2014;271(7):2049-60. doi: 10.1007/s00405-014-2914-9

9. Wittekindt D, Wittekindt C, Meissner W, Guntinas-Lichius O. Postoperative pain assessment after middle ear surgery. HNO 2012;60(11):974-84. doi: 10.1007/s00106-012-2556-4

10. Sommer M, Geurts JW, Stessel B, Kessels AG, Peters ML, Patijn J, van Kleef M, Kremer B, Marcus MA. Prevalence and predictors of postoperative pain after ear, nose and throat surgery. Arch Otolaryngol Head Neck Surg 2009;135(2):124-30. doi: 10.1001/archoto.2009.3

11. Kakehata S, Furukawa, T, Ito T, Kubota T, Futai K, Watanabe T. Comparison of Postoperative Pain in Patients Following Transcanal Endoscopic Versus Microscopic Ear Surgery. Otol Neurotol 2018;39(7):847-53. doi: 10.1097/MAO.0000000000001864

12. James AL. Endoscopic middle ear surgery in children. Otolaryngol Clin North Am 2013;46(2):233-44. doi:10.1016/j.otc.2012.10.007 